\title{
Contentious Feedback in Cellular Systems
}

\author{
Alex Reznik \\ InterDigital \\ King of Prussia, PA 19406 \\ Alex.Reznik@InterDigital.com
}

\author{
Eldad Zeira \\ InterDigital \\ Melville, NY, 11747 \\ Eldad.Zeira@InterDigital.com
}

\begin{abstract}
We consider the problem of counting mobile stations based on feedback they provide. For this problem we propose an anonymous contentious feedback mechanism which permits efficient counting and relate the resulting estimation problem to a well know combinatorial problem of "coupon collection." Using this relationship, we analyze the estimation problem in an idealized setting. We find the ML estimator and demonstrate that it is inappropriate in this setting. We then propose an estimator which is shown to achieve good performance over a large range of parameters.
\end{abstract}

\section{INTRODUCTION}

Consider the following situation: in a cellular system a number $(M)$ of mobile stations (MSs) need to provide feedback to the network. The feedback is single-bit and is used to indicate occurrence of some event. This is transmitted by sending a pre-defined signal when the event occurs. Absence of such a signal indicates non-occurrence of the event in question. A simple example of such a feedback is ACK/NACK associated with successful reception of a data packet (ACK) or a reception failure (NACK). In our case either an ACK or a NACK is sent explicitly, its opposite is indicated implicitly by its absence.

Our goal is to consider efficient methods for provisioning over-the-air signaling for such a feedback in the following scenario:

- The number of MSs $(M)$ is large enough that dedicating a feedback slot to each MS signal is not practical. In fact, the number of MSs may not be known at all and the goal of asking for feedback is to count MSs.

- It is not necessary to associate a received feedback signal with the MS that transmitted it - thus feedback is anonymous.

- The network/base station (BS) is interested in having a count of the number of MSs that sent feedback and not necessarily interested in correctly receiving each feedback signal.

These requirements suggest solving the problem by introducing a contentious and anonymous feedback channel which operates as follows:

- Feedback is requested within a particular time frame which is partitioned into $N$ slots.

- Whenever a MS needs to provide feedback (i.e. the appropriate triggering event has occurred at the MS) it randomly selects one of $N$ available slots and transmits a pre-defined signal in that slot. We could generalize the problem by making the MS transmit with probability $0<p<1$, however this does not change the essential nature of our results (except by scaling them by a factor of $\sim \frac{1}{p}$ ) and we omit the details for brevity.

- All feedback signals are identical - transmissions by multiple MSs in the same slot result in a collision.

- The predefined signal is designed in such a way that with very high probability signal collisions do not destroy each other. This is fairly easy - e.g. a constant signal modulated on the appropriate code, sub-carrier, etc. does the job in modern communication systems, such as CDMA and OFDM.

In this paper we make a further simplifying assumption:

- Signal collisions never destroy each other and the BS always accurately estimates whether a signal has been transmitted in a particular slot.

- Thus, the BS can accurately estimate how many of the $N$ slots were not used by any MSs in a particular frame, however if a slot has been used, the BS has no way of knowing how many MSs actually used it.

Under these assumptions a straightforward treatment of the problem as one of combinatorial counting and parameter estimation becomes possible. The results are presented in the paper, which is structured as follows. In Section 2 we discuss the underlying probability distributions and discuss the relevant properties of these. In particular we present a convergence property of the underlying distribution that is much stronger then the typical in probability convergence found in the literature. This is of particular importance in our context as it enables us to provide stronger guarantees in resource planning for a contentious feedback structure.

The Maximum Likelihood Estimator for $M$ is discussed in Section 3, where it is shown that it is a particularly bad estimator for this problem. In Section 4 we propose an alternative estimator. We demonstrate that this estimator works well in simulations. We conclude with some remarks in Section 5.

\section{The Probability Distributions}

Let $T$ be the number of used slots in any given frame. Thus, $T$ is an integer random variable with range $[0, \ldots, N]$. Our eventual goal is to estimate the value of $M$ from an observation $T$. To do so, we start by writing down the distribution of $T$ as parameterized by $M$ and $N$. This turns out to be a variant of the inverse coupon collector's problem [4],[6] and 
the distribution is given by

$P_{M}(t) \stackrel{\text { def }}{=} \operatorname{Pr}\{T=t\}=\left\{\begin{array}{cl}\frac{N !}{(N-t) !} \frac{S(M, t)}{N^{M}}, & 0 \leq t \leq \min (M, N) \\ 0, & \text { otherwise }\end{array}\right.$

where $S(M, t)$ is the Stirling Number of the Second Kind defined as [1], [5]

$$
S(M, t)=\frac{1}{t !} \sum_{j=0}^{t}(-1)^{(t-j)}\left(\begin{array}{l}
t \\
j
\end{array}\right) j^{M}
$$

The interested reader is referred to [1], [5] and references therein for properties of Stirling Numbers. Of these, we will use the following recursion:

$$
S(m, t)=t S(m, t-1)+S(m-1, t-1)
$$

The expected value of $T$ is easily found through the following argument. Each MS selects one of $N$ slots at random. Thus, the probability that some specific slot is not used is given by $\left(1-\frac{1}{N}\right)^{M}$. Let $\mathrm{I}(n)$ be the indicator function for slot $n: \mathrm{I}(n)=1$ if slot $n$ is used and $\mathrm{I}(n)=0$ otherwise. Then we have

$$
\begin{aligned}
\mathrm{E} T & =\sum_{n=1}^{N} \operatorname{Pr}\{\mathrm{I}(n)=1\} \\
& =N\left(1-\left(1-\frac{1}{N}\right)^{M}\right) \\
& \approx N\left(1-\exp \left(-\frac{M}{N}\right)\right)
\end{aligned}
$$

Another standard problem of interest to us is that of the stopping time in the coupon collector's problem. In our context, this is formulated as follows. Consider a frame with $N$ available slots. We have an infinite "supply" of MSs and these pick a slot for feedback in some sequential order. Fixing the number of slots, $N$, we define $W_{N}$ to be the index of the MS that picks the last unused slot (or equivalently $W$ is the number of MSs that provided feedback when the last slot became unavailable). The problem is a classical stopping time problem [2] and the stopping time distribution is given [3], [4]

$$
\operatorname{Pr}\left\{W_{N}=m\right\}=\frac{(N-1) !}{N^{m-1}} S(m-1, N-1)
$$

The expected value of $W_{N}$ can be computed without resorting to the explicit summation of (5) by using an elegant argument [6]. This is given by

$$
\mathrm{E} W_{N}=N \cdot H_{N}
$$

where $H_{N}$ is the $N^{\text {th }}$ Harmonic number:

$$
H_{N}=\sum_{k=1}^{N} \frac{1}{k}=\gamma+\ln N+\frac{1}{2 N}+o\left(\frac{1}{N}\right)
$$

where $\gamma \approx 0.577$ is the Euler-Mascheroni constant.

Using the expected value a weak in probability convergence result may be obtained using Markov's Inequality and this is often sufficient. However, as one of our concerns may be "planning" the number of slots made available per frame to support some potential maximal number of MSs we desire a stronger result. This is stated below

Lemma 1: For any fixed $\epsilon>0, W_{N} \leq N^{1+\epsilon} \ln N$ almost surely, i.e.

$$
\lim \sup _{N} \operatorname{Pr}\left\{W_{N} \geq N^{1+\epsilon} \ln N\right\}=0
$$

Proof: We prove the result using the Borel-Cantelli Lemma [2], which means that we must show that for any $\epsilon>0$

$$
\sum_{N} \operatorname{Pr}\left\{W_{N} \geq N^{1+\epsilon} \ln N\right\}<\infty
$$

We begin by defining some (non-stochastic) function $f(N)$ and expanding the definition of the Stirling Number in (5) to write

$$
\begin{aligned}
& \operatorname{Pr}\left\{W_{N} \geq f(N)\right\} \\
= & \sum_{m=f(N)}^{\infty} \sum_{j=0}^{N-1}(-1)^{j}\left(\begin{array}{c}
N-1 \\
j
\end{array}\right)\left(\frac{N-j-1}{N}\right)^{m-1} \\
= & \sum_{j=0}^{N-1}(-1)^{j}\left(\begin{array}{c}
N-1 \\
j
\end{array}\right) \sum_{m=f(N)}^{\infty}\left(\frac{N-j-1}{N}\right)^{m-1} \\
= & \sum_{j=0}^{N-1}(-1)^{j}\left(\begin{array}{c}
N \\
j+1
\end{array}\right)\left[1-\frac{j+1}{N}\right]^{(f(N)-1)}
\end{aligned}
$$

We then have

$$
\begin{aligned}
& \sum_{N=1}^{\infty} \operatorname{Pr}\left\{W_{N} \geq f(N)\right\} \\
= & \sum_{j=0}^{\infty} \sum_{N=j+1}^{\infty}(-1)^{j}\left(\begin{array}{c}
N \\
j+1
\end{array}\right)\left[1-\frac{j+1}{N}\right]^{(f(N)-1)}
\end{aligned}
$$

We shall look for a function $f(N)$ such that this series converges absolutely, i.e. we seek to make sure that

$$
\sum_{j=0}^{\infty} \sum_{N=j+1}^{\infty}\left(\begin{array}{c}
N \\
j+1
\end{array}\right)\left[1-\frac{j+1}{N}\right]^{(f(N)-1)}
$$

converges.

To do so, we set $f(N)-1=N^{1+\epsilon} \ln N$ and write

$$
\begin{aligned}
{\left[1-\frac{j+1}{N}\right]^{N^{1+\epsilon} \ln N} } & =\exp \left[N^{1+\epsilon} \ln N \ln \left(1-\frac{j+1}{N}\right)\right] \\
& \leq \exp \left[-\left(N^{\epsilon}(j+1)\right) \ln N\right] \\
& =N^{-\left(N^{\epsilon}(j+1)\right)}
\end{aligned}
$$

Using an upper bound on $\left(\begin{array}{c}N \\ j+1\end{array}\right) \leq \frac{N^{(j+1)}}{(j+1) !}$ we can then write

$$
\begin{aligned}
& \sum_{N=1}^{\infty} \operatorname{Pr}\left\{W_{N} \geq N \ln N+1\right\} \\
\leq & \sum_{j=0}^{\infty} \sum_{N=j+1}^{\infty}\left(\begin{array}{c}
N \\
j+1
\end{array}\right) N^{-\left(N^{\epsilon}(j+1)\right)} \\
\leq & \sum_{j=0}^{\infty} \frac{1}{(j+1) !} \sum_{N=j+1}^{\infty} N^{-\left(N^{\epsilon}-1\right)(j+1)}<\infty
\end{aligned}
$$


which completes the proof.

Lemma 1 is important in that it establishes that the expected value and maximal value of $W_{N}$ grow at roughly the same rate: $\mathrm{E} W_{N}=O(N \ln N)$, while $\mathrm{E} \sup \left(W_{N}\right)=$ $O\left(N^{1+\epsilon} \ln N\right)$. Since $\epsilon$ can be made arbitrarily small, given the maximal possible value of MSs, $M_{\max }$, a "safe" strategy for selecting the number of slots to allocate is to fix some constant $c$ and select $N$ such that

$$
M_{\max }=c N \ln N
$$

In Section 4 we demonstrate how this strategy works for even a fairly high constant: $c=2$.

Before moving on to analysis of estimator of $M$ we mention two properties which all "decent" estimators must satisfy. For any estimator $\hat{M}(t)$ we should have

$$
\begin{array}{ll}
\hat{M}(0) & =0 \\
\hat{M}(N) & \quad \text { undefined }
\end{array}
$$

The first property is easily proven - since a transmitting MS must use some slot, if no slots are used, the number of transmitting MSs must necessarily be 0 . The second property is based on the following intuition: if all the slots are filled we really have no way of figuring out which (presumably large) number of MSs is the most meaningful. For example, from an ML point of view, the larger the number of MSs the more likely it is that all slots are filled. The ML estimate when all slots are filled is therefore infinite. Keeping this in mind, we define our estimators only for $t \in\{1, \ldots, N-1\}$.

\section{The Maximum LiKelihood Estimator}

The maximum likelihood (ML) estimator $\hat{M}_{M L}$ turns out to be a poor estimator both theoretically and numerically. Specifically, we have

Lemma 2: As $N \rightarrow \infty, \hat{M}_{M L}(t) \rightarrow t$

Proof: The ML estimator is given by

$$
\hat{M}_{M L}(t)=\arg \max _{m} P_{m}(t)
$$

Isolating only those portions of (1) which depend on $M$ we define

$$
J_{t}(m) \stackrel{\text { def }}{=} \frac{S(m, t)}{N^{m}}
$$

and therefore $\hat{M}_{M L}(t)=\arg \max _{m} J_{t}(m)$.

Using (3) we can write

$$
J_{t}(m)=\frac{t}{N} J_{t}(m-1)+\frac{S(m-1, t-1)}{N^{m}}
$$

Observe now that $t \leq N$. Moreover, from (1) we have

$$
\frac{S(m-1, t-1)}{N^{m-1}} \frac{N !}{(N-t+1) !}<1
$$

and therefore

$$
\frac{S(m-1, t-1)}{N^{m}}<\frac{1}{N} \frac{(N-t+1) !}{N !} \leq \frac{1}{N}
$$

concluding that $\frac{S(m-1, t-1)}{N^{m}} \rightarrow 0 \forall t, m$ as $N \rightarrow \infty$. Thus, for large enough $N, J_{t}(m)<\frac{t}{N} J_{t}(m-1)$ and therefore $\hat{M}_{M L}$ is the smallest possible value of $m$, which is $m=t$.
This immediately gives us the following result stating the analytical problems of the ML estimator

Corollary 1: The asymptotic bias of the ML estimator and thus also its mean squared error is unbounded with $N$.

We illustrate the asymptotic failure of the ML estimator with some numerical results. Figure 1-4 show the ML estimator for $N=10,20,50$ and 100, respectively. The line $y(t)=t$ is plotted for reference. We observe that the estimator results in non-trivial (and in fact rather good) values for $N=10$ but begins to deteriorate as $N$ increase. At $N=100$ the ML estimate is essentially equal to $t$. In fact, it appears that a phase transition occurs for values of $t$ above about 32, at which point the ML estimator becomes approximately equal to $t$. At this point we do not have further intuition about this fact.

In some sense the result above makes intuitive sense. If the number of available slots is large and the observed number of used slots is small in comparison (i.e. $t<<N$ ), the probability of an MS choosing an used slot is quite low and the most likely single event is that each used slot is used by only a single MS. What is surprising and counter-intuitive is that for large $N$ the ML estimator sets $\hat{M}_{M L} \approx t$ even when $t$ approaches $N$.

\section{LOG-LinEAR ESTIMATION OF $M$}

We define an log-linear estimator for $M$ by inverting (4) to get

$$
\hat{M}_{L L}(t)=\frac{\log \left(1-\frac{t}{N}\right)}{\log \left(1-\frac{1}{N}\right)} \approx-N \ln \left(1-\frac{t}{N}\right)
$$

We note that the $\hat{M}_{L L}$ does not suffer from the deficiencies of the ML estimator. Its behavior is primarily determined by the ratio $\frac{t}{N}$ which figures in the numerator of (14). The denominator of (14) provides a scaling factor of approximately $N$ and does not impact the way the estimator varies with $t$.

The proposed estimator is not unbiased. To see this consider the case when $t=1$. Then $\hat{M}_{L L}=1$ - a clear bias. Nevertheless, Lemma 1 hints that a strong limit exists on the deviation of the actual value from the estimate - and this guarantee is confirmed by numerical results presented below.

We illustrate the numerical performance of this estimator in Figures 5-10. To generate these, we fix $N$ at 10, 50, 100 and determine the maximum value of $M$ by following Lemma 1 at $M=\lceil 2 N \ln N\rceil$. The resulting maximal values are 47, 392, 922. For each iteration, a value of $M$ is then chosen uniformly between 1 and the maximum and the test repeated over 100,000 iterations.

For each value of $N$ we present two results - a contour plot showing the number of occurrences of each $t, M$ pair (effectively an un-normalized joint distribution of the two). These plots also show the log-linear estimator. The second set of plots shows a normalized scatter plot of $\frac{M}{\hat{M}_{L L}(t)}$ versus $t$, which nicely illustrates the limited spread of $M$ around $\hat{M}_{L L}$.

\section{CONCLusions}

In this paper we considered efficient (in terms of airinterface resources) counting of MSs using feedback they provide. By casting the problem in an idealized setting, we 
showed that it is closely related to the well-known combinatorial problem of the coupon collector. In the context of estimation, we showed that the commonly used ML estimation approach is not appropriate for this problem. We then defined a log-linear estimator which we showed to perform quite well in simulations. Finally, using our strong almost sure convergence result on the stopping time problem, we provided guidelines for planning the number of feedback slots per frame that should be allocated in the system.

We note at this point that the idealized setting we adopted in this paper ignores several aspects of wireless communication systems. First, over-the-air interference and the resulting errors are ignored. More importantly, we completely ignore the possibility of refining our estimate using the actual amount of received power per access slot. Both of these are important areas of further research in this area.

\section{REFERENCES}

[1] R. P. Stanley. Enumerative Combinatorics, Vol. 1. Cambridge U. Press, Cambridge UK, 1997.

[2] P. Billingsley. Probability and Measure, $3^{\text {rd }}$ Ed., Wiley-Interscience, New York, 1995.

[3] W. Feller. An Introduction to Probability Theory and Its Applications, Vol. 1, $3^{\text {rd }}$ Ed., John Wiley \& Sons, New York, 1968.

[4] B. Dawkins. "Siobhan's Problem: The Coupon Collector Revisited," The American Statistician, vol. 45, no. 1, pp. 76-82, Feb. 1991.

[5] E. W. Weisstein. "Stirling Number of the Second Kind," MathWorld-A Wolfram Web Resource. http://mathworld.wolfram.com/ StirlingNumberofthesecondKind.html

[6] "Coupon Collector's Problem," Wikipedia.

http://en.wikipedia.org/wiki/Coupon_collector's_problem

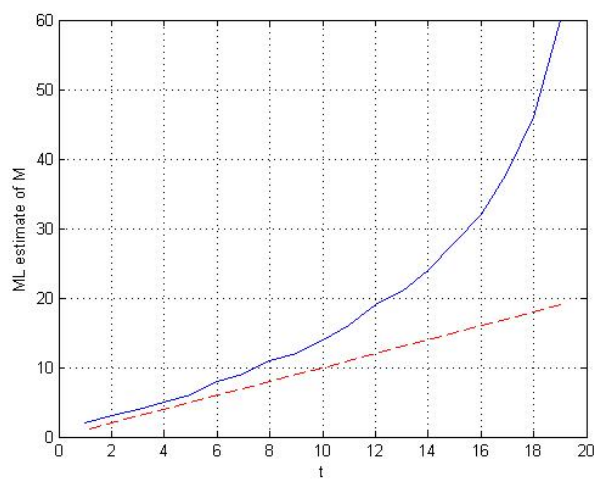

Fig. 2. ML estimator for $N=20$

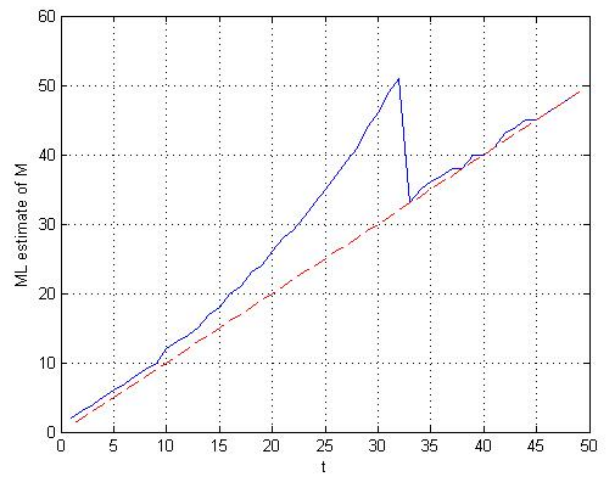

Fig. 3. ML estimator for $N=50$

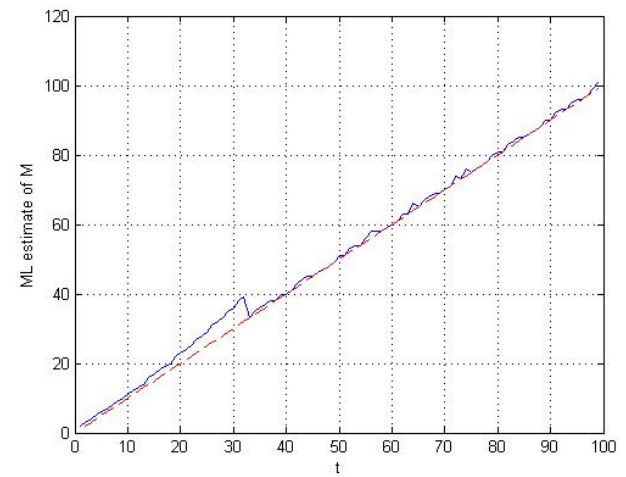

Fig. 4. ML estimator for $N=100$

Fig. 1. ML estimator for $N=10$ 


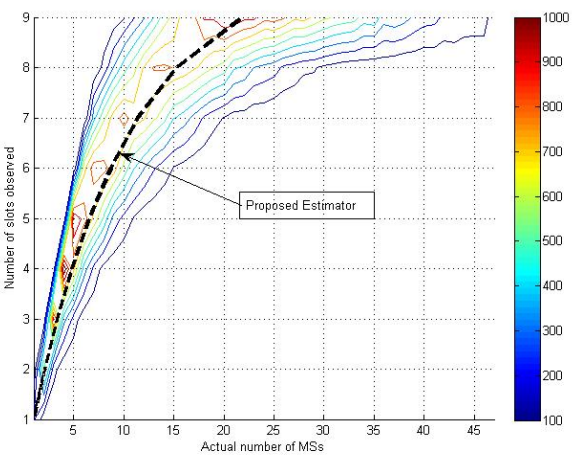

Fig. 5. $(t, M)$ Contour and LL estimator for $N=10$

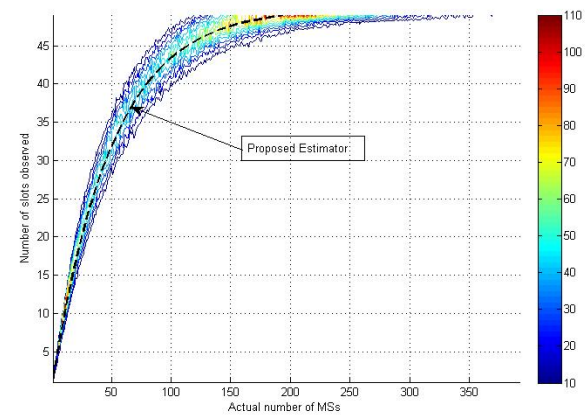

Fig. 6. $(t, M)$ Contour and LL estimator for $N=50$

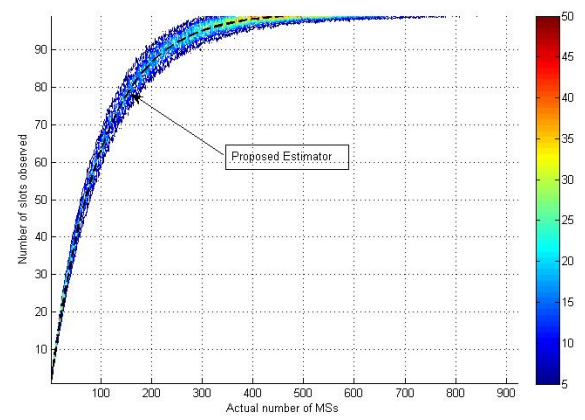

Fig. 7. $(t, M)$ Contour and LL estimator for $N=100$

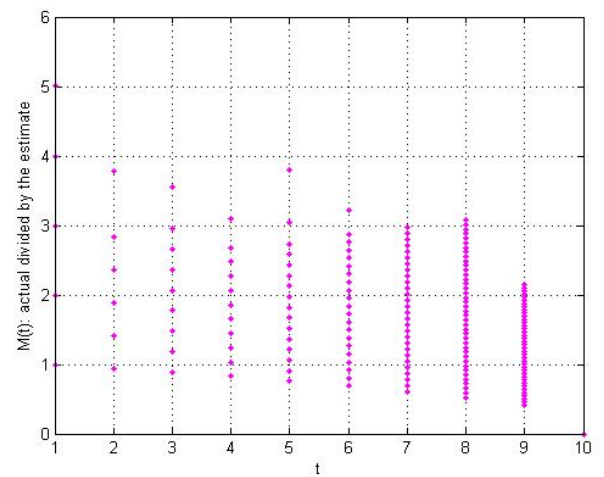

Fig. 8. Normalized $(t, M)$ scatter for $N=10$

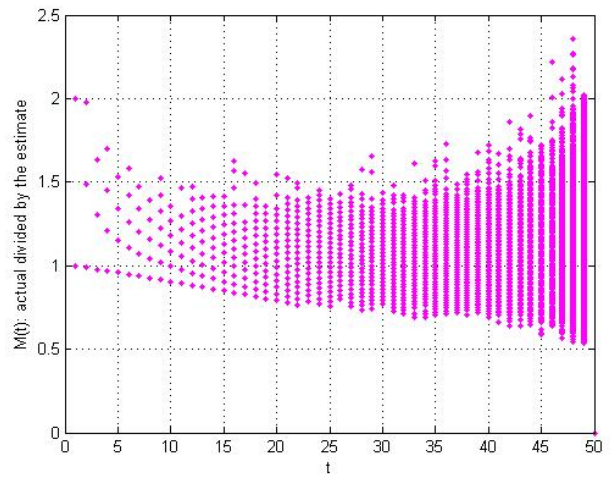

Fig. 9. Normalized $(t, M)$ scatter for $N=50$

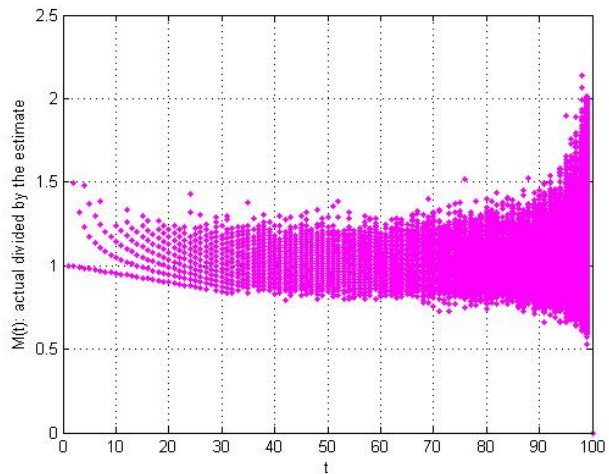

Fig. 10. Normalized $(t, M)$ scatter for $N=100$ 